\title{
Adaptive MIMO Supervisory Control Design Using Modeling Error
}

\author{
Zhi-Ren Tsai $^{1,2}$ and Yau-Zen Chang ${ }^{3}$ \\ ${ }^{1}$ Department of Computer Science \& Information Engineering, Asia University, Wufeng District, Taichung 41354, Taiwan \\ ${ }^{2}$ Department of Medical Research, China Medical University Hospital, China Medical University, Taichung 40402, Taiwan \\ ${ }^{3}$ Department of Mechanical Engineering, Chang Gung University, Taoyuan 33302, Taiwan
}

Correspondence should be addressed to Yau-Zen Chang; zen@mail.cgu.edu.tw

Received 14 July 2015; Revised 14 September 2015; Accepted 15 September 2015

Academic Editor: Rongwei Guo

Copyright (C) 2015 Z.-R. Tsai and Y.-Z. Chang. This is an open access article distributed under the Creative Commons Attribution License, which permits unrestricted use, distribution, and reproduction in any medium, provided the original work is properly cited.

\begin{abstract}
This paper proposes an adaptive control scheme for nonlinear systems with significant nonminimum phase dynamics. The scheme is composed of an inner-level adaptive fuzzy PD control law and an outer-level supervisory control law. Importantly, the inner-level controller of the two-level scheme is designed based on a fuzzy model, which takes nonminimum phase phenomenon and modeling error explicitly into account. The scheme is both much simpler in design and more applicable to general nonlinear systems when compared with most existing nonlinear controllers. Effectiveness of the proposed control strategy is demonstrated by numerical simulation of the control of a five-degree-of-freedom aircraft system in the face of bursting disturbances.
\end{abstract}

\section{Introduction}

Many critical dynamic systems, such as aircraft, are nonminimum phase, MIMO, and highly nonlinear, which undergo significant disturbances and parameter variation during operation. To control these systems, robust control [1], optimal tuning of fuzzy controllers with output sensitivity function [2], adaptive control [3-5], and feedback linearization with discrete sliding-mode control [6] have attracted much attention from both academic and industrial communities due to their robustness to uncertainties. Recently, many interests have been focused on applying these techniques to flight control systems, such as $[7,8]$. However, for systems with significant nonminimum phase phenomenon, direct application of these approaches tends to introduce unstable zero dynamics.

For instance, in [9], the nonminimum phase plants are approximated by minimum phase models. The research [10] applied the output regulation theory to solve the output tracking problem, but a set of partial differential equations must be solved. The control scheme of [11] is based on decomposing the aircraft dynamics into a minimum phase part and a nonminimum phase part. Inversion is used on the minimum phase part to obtain asymptotic tracking, while a robust linear control approach is used to stabilize the nonminimum phase part, which is linearized at equilibrium. As this strategy is based on local linearization of the nonminimum phase part, the result can only apply to simplified models.

By estimating parameters online, adaptive control can adapt to a controlled system with varying or unknown parameters. Nevertheless, in spite of the prosperous literature of adaptive control, practical application of these control strategies on MIMO systems has been restricted by the lack of assurance in closed-loop stability. Among them, the adaptive neural controller of [3] is too complex to implement, while the adaptive fuzzy terminal sliding-mode controller of $[4,5]$ is applicable only to robotic manipulators.

The proposed adaptive control scheme is inspired by [12], which was developed for SISO nonlinear systems based on the feedback linearization technique, with the distinction that the scheme is extended to nonminimum phase MIMO control systems.

The scheme is composed of an inner-level tracking control law and an outer-level supervisory control law. 
The design procedure is hence divided into two parts. First, an adaptive fuzzy-model-based PD control scheme is designed at the inner level to achieve robust output tracking. Special care is taken for the nonminimum phase fuzzy subsets in the control law by restricting parameter magnitudes in the singular-value decomposition operation. Next, a supervisory controller is employed at the outer level to minimize both the approximation error between the fuzzy model and the plant and the effects of external disturbance. Effectiveness of the adaptive control scheme is demonstrated by simulation results of the fight control of a complete 5-DOF aircraft model.

\section{Problem Formulation}

System dynamics of the plant are firstly represented in a general MIMO state-space representation as

$$
\begin{aligned}
& \dot{x}=F(x)+G(x) \cdot u+w_{0}, \\
& y=H \cdot x,
\end{aligned}
$$

where $x \in \mathfrak{R}^{n \times 1}$ is the state vector, $u \in \mathfrak{R}^{m \times 1}$ is the control vector, $w_{0} \in \mathfrak{R}^{n \times 1}$ is the disturbance vector, $y \in \mathfrak{R}^{N \times 1}$ is the output vector, and $F, G$ are corresponding nonlinear matrices in state vectors with $H$ being a constant matrix, all of compatible dimensions.

Equation (1) can be further represented in output vector $y$ as

$$
\begin{aligned}
\dot{y}= & H \cdot F(x)+H \cdot G(x) \cdot u+H \cdot w_{0} \\
= & f(x)+g(x) \cdot u+w \\
= & h_{f} \cdot A_{f}+\sum_{i=1}^{L} h_{i} \cdot B_{i} \cdot u+\left[f(x)-h_{f} \cdot A_{f}\right] \\
& +\left[g(x)-\sum_{i=1}^{L} h_{i} \cdot B_{i}\right] \cdot u+w \\
= & h_{f} \cdot A_{f}+\sum_{i=1}^{L} h_{i} \cdot B_{i} \cdot u+\bar{e}_{\bmod } \\
\triangleq & h_{f} \cdot A_{f}+B_{c} \cdot u+\bar{e}_{\bmod },
\end{aligned}
$$

where

$$
\begin{aligned}
B_{c} \triangleq & \sum_{i=1}^{L} h_{i} \cdot B_{i}, \\
h_{f} & =\left[\begin{array}{cccc}
h_{f 1} & 0 & 0 & 0 \\
0 & h_{f 2} & 0 & 0 \\
0 & 0 & \ddots & 0 \\
0 & 0 & 0 & h_{f n}
\end{array}\right],
\end{aligned}
$$

$$
\begin{aligned}
A_{f}= & {\left[\begin{array}{llll}
A_{f 1} & A_{f 2} & \cdots & A_{f n}
\end{array}\right]^{T}, } \\
f_{1}= & \sum_{i=1}^{p} h_{1 i} \cdot A_{1 i}=h_{f 1} \cdot A_{f 1}, \\
f_{2}= & \sum_{i=1}^{p} h_{2 i} \cdot A_{2 i}=h_{f 2} \cdot A_{f 2}, \ldots, \\
f_{n}= & \sum_{i=1}^{p} h_{n i} \cdot A_{n i}=h_{f n} \cdot A_{f n}, \\
\bar{e}_{\text {mod }}= & {\left[f(x)-h_{f} \cdot A_{f}\right]+\left[g(x)-\sum_{i=1}^{L} h_{i} \cdot B_{i}\right] \cdot u } \\
& +w,
\end{aligned}
$$

and the external disturbance $w=H \cdot w_{0}$.

In the last representation, it is assumed that $g(x)$ is bounded and is away from singularity in a compact set. Furthermore, $f(x)$ and $g(x)$ are identified in fuzzy form as $h_{f}(y) \cdot A_{f}(t)$ and $\sum_{j=1}^{L} h_{j}(y) \cdot B_{j}$, respectively, where the fuzzy logic systems are universal approximations which can uniformly approximate nonlinear continuous functions to arbitrary accuracy [13-15].

\section{Controller Design for the Nonminimum Phase Dynamics}

Firstly, the tracking error is defined as

$$
e(t)=-\bar{e}
$$

where $\bar{e}=\left(y_{r}-y\right)\left(y_{r}\right.$ is reference input $)$; we have that

$$
\begin{aligned}
\dot{e}(t) & =h_{f} \cdot A_{f}+\sum_{i=1}^{L} h_{i} \cdot B_{i} \cdot u+\bar{e}_{\mathrm{mod}}-\dot{y}_{r} \\
& =h_{f} \cdot A_{f}+B_{c} \cdot u+\bar{e}_{\mathrm{mod}}-\dot{y}_{r}
\end{aligned}
$$

where $u$ is a combination of two signals [16]:

$$
u=u_{F}+u_{S}
$$

with

$$
\begin{aligned}
u_{F}= & \left(1-I^{*}\right) \cdot B_{c}^{-1} \\
& \cdot\left\{-h_{f}(y) \cdot A_{f}(t)+\dot{y}_{r}+K_{P} \cdot e(t)+K_{D} \cdot \dot{e}(t)\right\}, \\
u_{S}= & -I^{*} \cdot \operatorname{sgn}\left(B_{c} \cdot P \cdot e(t)\right) \\
& \cdot\left\{\left|B_{c}^{-1} \cdot\left[h_{f}(y) \cdot A_{f}(t)-\dot{y}_{r}\right]\right|+e_{U}\right\} .
\end{aligned}
$$




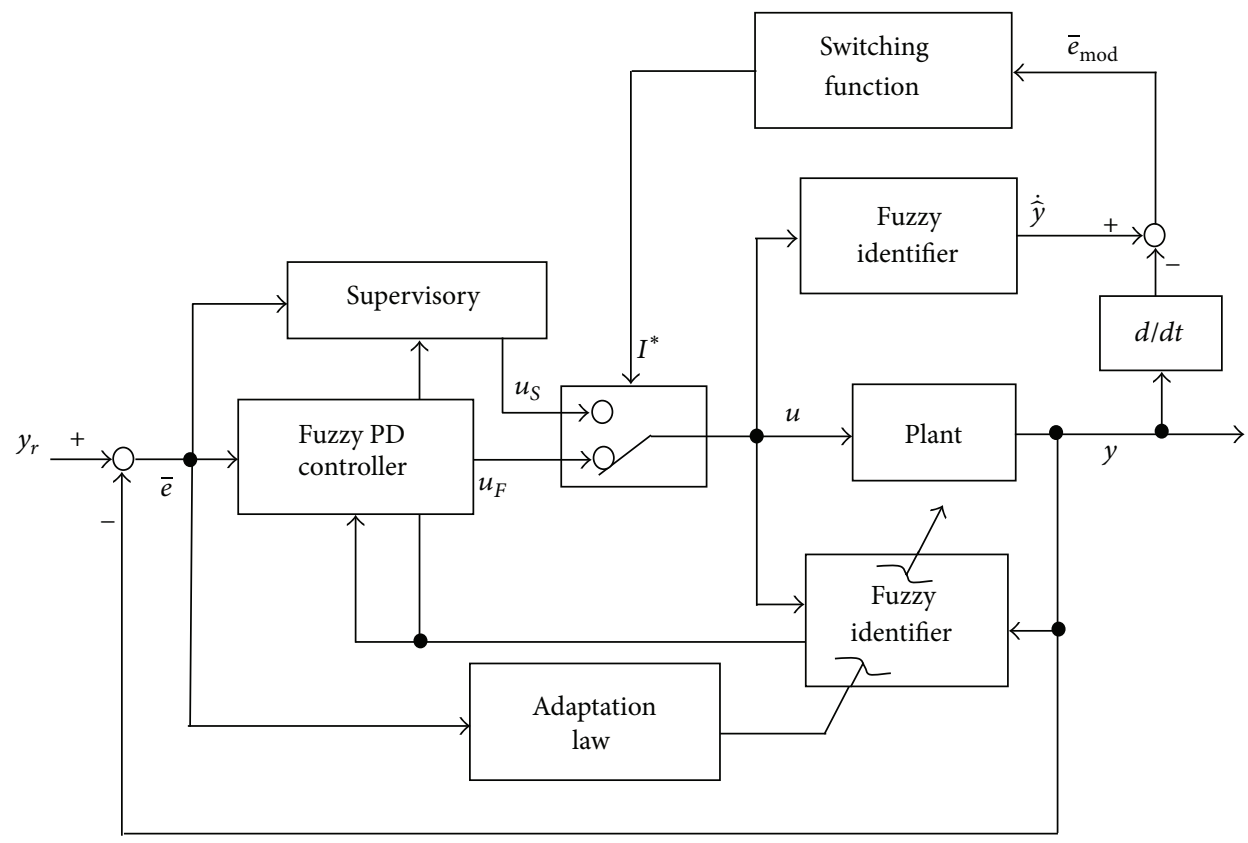

FIGURE 1: The proposed two-level switching control scheme.

In (7), the proportional gain of the inner fuzzy control law is designed as

$$
K_{P}=\alpha-R^{-1} \cdot P
$$

The switching variable in both (7) and (8) is defined as

$$
\begin{aligned}
& I^{*}=0, \text { if }\left\|\bar{e}_{\text {mod }}\right\| \leq \bar{e}_{U}, \\
& I^{*}=1, \quad \text { otherwise, }
\end{aligned}
$$

with

$$
\begin{aligned}
\left|B_{c}^{-1} \cdot \bar{e}_{\mathrm{mod}}\right| & \leq e_{U}, \\
\alpha & =\left[\begin{array}{cccc}
-\alpha_{1} & 0 & 0 & 0 \\
0 & -\alpha_{2} & 0 & 0 \\
0 & 0 & \ddots & 0 \\
0 & 0 & 0 & -\alpha_{n}
\end{array}\right], \\
P & >0 \\
R & >0 .
\end{aligned}
$$

A complete control scheme of the two-level architecture is shown in Figure 1.

To avoid encountering singularity of the control law, the singular-value decomposition of the matrix $B_{c}$ is introduced as follows:

$$
B_{c}=U \cdot S \cdot V^{T}
$$

where

$$
S=\left[\begin{array}{cccc}
\sigma_{1} & 0 & 0 & 0 \\
0 & \sigma_{2} & 0 & 0 \\
0 & 0 & \ddots & 0 \\
0 & 0 & 0 & \sigma_{n}
\end{array}\right]
$$

and $\sigma_{i}$ is replaced by $\varepsilon$ if $\sigma_{i} \leq \varepsilon$, where $\varepsilon$ is a small value.

Substituting the adaptive fuzzy PD controller $u_{F}$ (7) into (5), we have

$$
\dot{e}(t)=K_{P} \cdot e(t)+h_{f}(y) \cdot \widetilde{A}_{f}(t)+e_{\mathrm{mod}}
$$

after algebra manipulations, where

$$
\widetilde{A}_{f}(t)=A_{f}^{*}-A_{f}(t)
$$

and the modeling error

$$
\begin{aligned}
e_{\bmod }= & {\left[f(x)-h_{f}(y) \cdot A_{f}^{*}\right] } \\
& +\left[g(x)-\sum_{i=1}^{L} h_{i}(y) \cdot B_{i}\right] \cdot u+w+K_{D} \\
& \cdot \dot{e}(t) .
\end{aligned}
$$


In the following derivation, we need the following condition to be satisfied [17-20]:

$$
J \leq e(0)^{T} \cdot P \cdot e(0)+\gamma_{f}^{-1} \cdot \operatorname{trace}\left(\widetilde{A}_{f}(0)^{T} \cdot \widetilde{A}_{f}(0)\right),
$$

where

$$
\begin{aligned}
J= & \int_{0}^{t_{f}}\left[e(t)^{T} \cdot\left(Q+P^{T} \cdot R^{-T} \cdot P\right) \cdot e(t)-\rho^{2} \cdot e_{\bmod }^{T}\right. \\
& \left.\cdot e_{\mathrm{mod}}\right] \cdot d t
\end{aligned}
$$

the weighting factor $\gamma_{f}>0, \rho^{2} \cdot I \geq R$, the matrix $Q>$ 0 , and $\|X\|_{F}=\sqrt{\operatorname{trace}\left(X^{T} \cdot X\right)}$ is the Frobenius norm of matrix $X$. Derivation of this condition, (17), is given in the Appendix.

Hence, we have that

$$
\begin{aligned}
& \dot{\widetilde{A}}_{f}(t)=-\dot{A}_{f}=-\gamma_{f} \cdot h_{f}(y)^{T} \cdot P \cdot e(t), \\
& {\left[\alpha^{T} \cdot P+P \cdot \alpha+Q-P^{T} \cdot R^{-T} \cdot P\right]=-\rho^{-2} \cdot P^{T} \cdot P .}
\end{aligned}
$$

Furthermore, to guarantee boundedness of $A_{f}$, the parameter update laws must be modified as follows:

$$
\dot{A}_{f}= \begin{cases}\gamma_{f} \cdot h_{f}(y)^{T} \cdot P \cdot e(t), & \text { if }\left\|A_{f}\right\|<M_{f} \text { or }\left(\left\|A_{f}\right\|=M_{f}, \dot{A}_{f}^{T} \cdot A_{f} \leq 0\right), \\ F\left(\gamma_{f} \cdot h_{f}(y)^{T} \cdot P \cdot e(t)\right), & \text { otherwise, }\end{cases}
$$

where $M_{f}$ is a positive design parameter and the projection function $F(\cdot)$ is defined as

$$
\begin{aligned}
F\left(\gamma_{f} \cdot h_{f}(y)^{T} \cdot P \cdot e(t)\right) & \\
= & \gamma_{f} \cdot h_{f}(y)^{T} \cdot P \cdot e(t)-\gamma_{f} \\
& \cdot \frac{A_{f} \cdot A_{f}^{T} \cdot h_{f}(y)^{T} \cdot P \cdot e(t)}{\left\|A_{f}\right\|^{2}} .
\end{aligned}
$$

Next, the supervisor control law of (8) is designed by the following Lyapunov candidate:

$$
V=e(t)^{T} \cdot P \cdot e(t) .
$$

Its time derivative, $\dot{V}$, can be obtained as

$$
\begin{aligned}
\dot{V}= & {\left[h_{f} \cdot A_{f}+B_{c} \cdot u_{S}+\bar{e}_{\mathrm{mod}}-\dot{y}_{r}\right]^{T} \cdot P \cdot e(t) } \\
& +e(t)^{T} \cdot P \cdot\left[h_{f} \cdot A_{f}+B_{c} \cdot u_{S}+\bar{e}_{\mathrm{mod}}-\dot{y}_{r}\right] \\
= & 2 e(t)^{T} \cdot P \cdot B_{c} \cdot u_{S}+2 e(t)^{T} \cdot P \\
& \cdot\left[h_{f} \cdot A_{f}+\bar{e}_{\mathrm{mod}}-\dot{y}_{r}\right] .
\end{aligned}
$$

Substituting (8) into (23) yields

$$
\begin{gathered}
\dot{V} \leq 2 e(t)^{T} \cdot P \cdot B_{c} \cdot u_{S}+2\left|e(t)^{T} \cdot P \cdot B_{c}\right| \\
\cdot\left|B_{c}^{-1} \cdot\left(h_{f} \cdot A_{f}+\bar{e}_{\mathrm{mod}}-\dot{y}_{r}\right)\right| \leq 0 .
\end{gathered}
$$

Hence, we can infer that if the supervisory control signal (8) is injected into fuzzy system (2), time derivative of the Lyapunov candidate $\dot{V} \leq 0$ and system (2) is UUB stable.

\section{Numerical Simulation}

In this section, the proposed control strategy is applied on a five-degree-of-freedom aircraft system described in [21] for performance evaluation. We consider the angle of attack $\alpha$ and the roll angle $\phi$ as outputs to be tracked. Tracking of angle of attack is directly related to tracking of normal acceleration [21], which plays an important role in many practical maneuvers.

Let $b=3$ be the reference length $(\mathrm{m}), \bar{c}=2$ the mean aerodynamic chord $(\mathrm{m}), g=9.8$ the gravitational acceleration $\left(\mathrm{m} / \mathrm{s}^{2}\right), I=50$ the moment of inertia $\left(\mathrm{kg}-\mathrm{m}^{2}\right), p$ the roll angle rate, $q$ the pitch angle rate, $r$ the yaw angle rate, $Q=80$ the dynamic pressure $\left(\mathrm{kg} / \mathrm{m}^{2}\right), S=5$ the reference wing area $\left(\mathrm{m}^{2}\right), V=100$ the aircraft velocity $(\mathrm{m} / \mathrm{s}), \theta$ the pitch angle, $\delta_{a}=0$ the aileron deflection, $\delta_{e}$ the elevator deflection, $\delta_{r}$ the rudder deflection, and $m=100$ the mass of aircraft (kg); the aircraft dynamics can be written as [21]

$$
\left[\begin{array}{c}
\dot{p} \\
\dot{q} \\
\dot{r}
\end{array}\right]=\left[\begin{array}{c}
f_{p} \\
f_{q} \\
f_{r}
\end{array}\right]+\left[\begin{array}{ccc}
L_{\delta a} & 0 & L_{\delta r} \\
0 & M_{\delta e} & 0 \\
N_{\delta a} & 0 & N_{\delta r}
\end{array}\right] \cdot\left[\begin{array}{c}
\delta_{a} \\
\delta_{e} \\
\delta_{r}
\end{array}\right],
$$$$
\left[\begin{array}{c}
\dot{\alpha} \\
\dot{\beta} \\
\dot{\varphi} \\
\dot{\theta}
\end{array}\right]=\left[\begin{array}{c}
f_{\alpha} \\
f_{\beta} \\
0 \\
0
\end{array}\right]+\left[\begin{array}{ccc}
-t_{\beta} \cdot c_{\alpha} & 1 & -t_{\beta} \cdot s_{\alpha} \\
s_{\alpha} & 0 & -c_{\alpha} \\
1 & t_{\theta} \cdot s_{\varphi} & t_{\theta} \cdot c_{\varphi} \\
0 & c_{\varphi} & -s_{\varphi}
\end{array}\right] \cdot\left[\begin{array}{l}
p \\
q \\
r
\end{array}\right]
$$ 
where

$$
\begin{aligned}
& \phi=y_{1}, \\
& \alpha=y_{2}, \\
& L_{\delta a}=I \cdot Q \cdot S \cdot b \cdot C_{l \delta a}, \\
& L_{\delta r}=I \cdot Q \cdot S \cdot b \cdot C_{l \delta r}, \\
& M_{\delta e}=I \cdot Q \cdot S \cdot \bar{c} \cdot C_{m \delta e} \text {, } \\
& N_{\delta a}=I \cdot Q \cdot S \cdot b \cdot C_{n \delta a} \text {, } \\
& N_{\delta r}=I \cdot Q \cdot S \cdot b \cdot C_{n \delta r} \text {, } \\
& f_{p}=\frac{Q \cdot S \cdot I \cdot b^{2} \cdot C_{l p} \cdot p}{2 V}, \\
& f_{q}=\frac{Q \cdot S \cdot I \cdot \bar{c}^{2} \cdot C_{m q} \cdot q}{2 V}, \\
& f_{r}=\frac{Q \cdot S \cdot I \cdot b^{2} \cdot C_{n r} \cdot r}{2 V}, \\
& f_{\alpha}=\frac{-Q \cdot S \cdot C_{L \alpha} \cdot \alpha+m \cdot g \cdot\left(c_{\theta} \cdot c_{\phi} \cdot c_{\alpha}+s_{\theta} \cdot s_{\alpha}\right)}{m \cdot V \cdot c_{\beta}}, \\
& f_{\beta}=\frac{Q \cdot S \cdot C_{Y \beta} \cdot \beta+m \cdot g \cdot\left[s_{\theta} \cdot c_{\alpha} \cdot s_{\beta}+c_{\theta} \cdot s_{\phi} \cdot c_{\beta}-c_{\theta} \cdot c_{\phi} \cdot s_{\alpha} \cdot s_{\beta}\right]}{m \cdot V}, \\
& t_{\beta}=\tan (\beta), \\
& s_{\beta}=\sin (\beta) \text {, } \\
& c_{\beta}=\cos (\beta), \\
& s_{\theta}=\sin (\theta), \\
& c_{\theta}=\cos (\theta), \\
& t_{\theta}=\tan (\theta) \text {, } \\
& c_{\alpha}=\cos (\alpha) \text {, } \\
& s_{\alpha}=\sin (\alpha) \text {, } \\
& s_{\phi}=\sin (\phi) \text {, } \\
& c_{\phi}=\cos (\phi) \text {. }
\end{aligned}
$$

In the following simulation, we assume

$$
\begin{aligned}
C_{l \delta a} & =-10^{-4}, \\
C_{l \delta r} & =10^{-2}, \\
C_{m \delta e} & =-1.6 \times 10^{-4},
\end{aligned}
$$

$$
\begin{aligned}
& C_{n \delta a}=10^{-2}, \\
& C_{n \delta r}=-10^{-4}, \\
& C_{l p}=-3.8 \times 10^{-2}, \\
& C_{m q}=-0.9 \times 10^{-2},
\end{aligned}
$$




$$
\begin{aligned}
& C_{n r}=-4.5 \times 10^{-3}, \\
& C_{L \alpha}=2.8 \times 10^{-1}, \\
& C_{Y \beta}=-2.8 .
\end{aligned}
$$

For the inner fuzzy control law, we select the following membership functions:

$$
\begin{aligned}
& \mu_{F_{1}^{1}}\left(y_{1}\right)=\exp \left[-\frac{1}{2}\left(\frac{y_{1}-c_{11}}{d_{11}}\right)^{2}\right], \\
& \mu_{F_{1}^{2}}\left(y_{1}\right)=\exp \left[-\frac{1}{2}\left(\frac{y_{1}-c_{12}}{d_{12}}\right)^{2}\right], \\
& \mu_{F_{1}^{3}}\left(y_{1}\right)=\exp \left[-\frac{1}{2}\left(\frac{y_{1}-c_{13}}{d_{13}}\right)^{2}\right], \\
& \mu_{F_{1}^{4}}\left(y_{1}\right)=\exp \left[-\frac{1}{2}\left(\frac{y_{1}-c_{14}}{d_{14}}\right)^{2}\right], \\
& \mu_{F_{2}^{1}}\left(y_{2}\right)=\exp \left[-\frac{1}{2}\left(\frac{y_{2}-c_{21}}{d_{21}}\right)^{2}\right], \\
& \mu_{F_{2}^{2}}\left(y_{2}\right)=\exp \left[-\frac{1}{2}\left(\frac{y_{2}-c_{22}}{d_{22}}\right)^{2}\right], \\
& \mu_{F_{2}^{3}}\left(y_{2}\right)=\exp \left[-\frac{1}{2}\left(\frac{y_{2}-c_{23}}{d_{23}}\right)^{2}\right], \\
& \mu_{F_{2}^{4}}\left(y_{2}\right)=\exp \left[-\frac{1}{2}\left(\frac{y_{2}-c_{24}}{d_{24}}\right)^{2}\right],
\end{aligned}
$$

where

$$
\begin{aligned}
& c_{11}=c_{21}=0, \\
& c_{12}=c_{22}=0.4, \\
& c_{13}=c_{23}=0.8, \\
& c_{14}=c_{24}=1.2, \\
& d_{11}=d_{21}=d_{12}=d_{22}=d_{13}=d_{23}=d_{14}=d_{24}=0.4 .
\end{aligned}
$$

Furthermore, 8 fuzzy rules of the following form comprise the fuzzy rule base:

$$
\begin{aligned}
& R^{(1)}: \text { if } y_{1} \text { is } F_{1}^{j} \text {, then } f_{1}=A_{1 j} \text { for } j=1,2,3,4 \text { and } \\
& l=1,2,3,4 . \\
& R^{(2)}: \text { if } y_{2} \text { is } F_{2}^{j} \text {, then } f_{2}=A_{2 j} \text { for } j=1,2,3,4 \text { and } \\
& l=5,6,7,8 .
\end{aligned}
$$
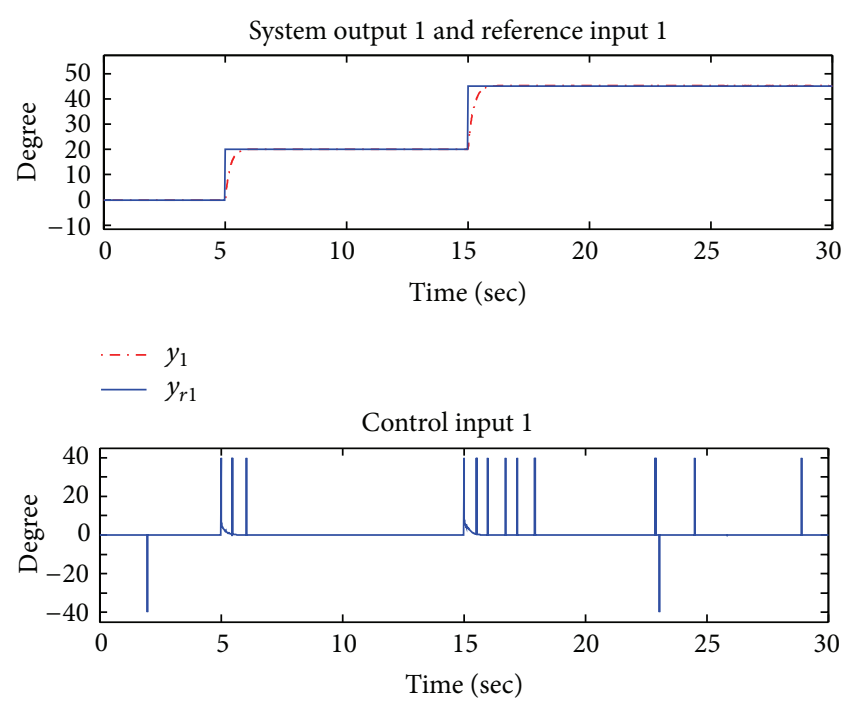

FIGURE 2: Time history of system output $y_{1}$ (the angle of attack, $\alpha$ ), reference $y_{r 1}$, and control input $u_{1}$.

Then, we obtain the following initial system parameters:

$$
\begin{aligned}
& A_{11}=-0.1338, \\
& A_{12}=0.5183, \\
& A_{13}=-1.0891, \\
& A_{14}=1.546, \\
& A_{21}=-1.0328, \\
& A_{22}=1.669, \\
& A_{23}=-0.0957, \\
& A_{24}=-0.3342,
\end{aligned}
$$

$$
B_{c}=\left[\begin{array}{cc}
16.5381 & -0.4429 \\
1.1439 & 0.4489
\end{array}\right] \text {. }
$$

Finally, we design the following control gains:

$$
\begin{aligned}
K_{P} & =\left[\begin{array}{cc}
-0.52 & 0 \\
0 & -51
\end{array}\right], \\
K_{D} & =\left[\begin{array}{cc}
-0.05 & 0 \\
0 & -4.9
\end{array}\right] .
\end{aligned}
$$

The tracking performances of $\alpha$ and $\phi$, together with the reference (or command), are presented in Figures 2 and 3. These figures show the responses with several step reference inputs. The disturbance is $w=[1,1,1,1]^{T} \cdot \delta(t-2)$, a burst at $t=2 \mathrm{~s}$.

From the simulation results, it is clear that the output tracks the desired command asymptotically with small transient errors, and the zero dynamics remain stable for all the simulated interval. 


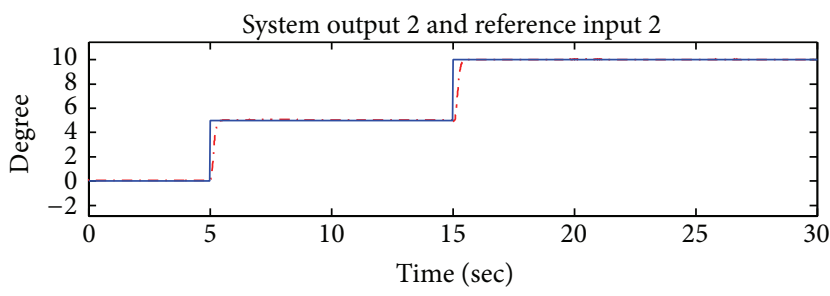

$\cdots y_{2}$

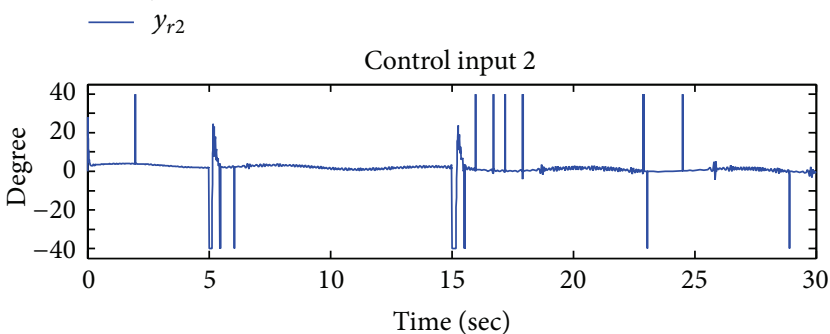

Figure 3: Time history of system output $y_{2}$ (the roll angle, $\phi$ ), reference $y_{r 2}$, and control input $u_{2}$.

\section{Conclusion}

We propose a two-level adaptive control scheme for nonlinear systems, such as the aircraft, which are MIMO and suffer from nonminimum phase phenomena. The control scheme is composed of an inner-level adaptive fuzzy PD control law and an outer-level supervisory control law. Importantly, the outerlevel controller of the two-level scheme is designed based on a fuzzy model taking nonminimum phase phenomena and modeling error explicitly into account. Special care is taken of the nonminimum phase fuzzy subsets by restricting the magnitude of parameters in the singular-value decomposition operation.

The control strategy is much simpler and applicable to general MIMO, nonlinear, and nonminimum phase systems when compared with [3-5]. Simulation results of the application of the proposed control scheme on a five-degree-offreedom nonlinear aircraft model verify its effectiveness.

\section{Appendix}

\section{Derivation of the Condition of (17)}

Consider

$$
\begin{aligned}
J= & \int_{0}^{t_{f}}\left[e(t)^{T} \cdot\left(Q+P^{T} \cdot R^{-T} \cdot P\right) \cdot e(t)-\rho^{2} \cdot e_{\mathrm{mod}}^{T}\right. \\
& \left.\cdot e_{\mathrm{mod}}\right] \cdot d t=e(0)^{T} \cdot P \cdot e(0)-e\left(t_{f}\right)^{T} \cdot P \\
& \cdot e\left(t_{f}\right)+\gamma_{f}^{-1} \cdot \operatorname{trace}\left(\widetilde{A}_{f}(0)^{T} \cdot \widetilde{A}_{f}(0)\right)-\gamma_{f}^{-1} \\
& \cdot \operatorname{trace}\left(\widetilde{A}_{f}\left(t_{f}\right)^{T} \cdot \widetilde{A}_{f}\left(t_{f}\right)\right)+\int_{0}^{t_{f}}\left[e(t)^{T} \cdot(Q\right. \\
& \left.+P^{T} \cdot R^{-T} \cdot P\right) \cdot e(t)-\rho^{2} \cdot e_{\bmod }^{T} \cdot e_{\bmod }+\dot{e}(t)^{T}
\end{aligned}
$$

$$
\begin{aligned}
& \cdot P \cdot e(t)+e(t)^{T} \cdot P \cdot \dot{e}(t)+\gamma_{f}^{-1} \cdot \operatorname{trace}\left(\dot{\vec{A}}_{f}(t)^{T}\right. \\
& \left.\left.\cdot \widetilde{A}_{f}(t)\right)+\gamma_{f}^{-1} \cdot \operatorname{trace}\left(\widetilde{A}_{f}(t)^{T} \cdot \dot{\widetilde{A}}_{f}(t)\right)\right] d t \\
& \leq e(0)^{T} \cdot P \cdot e(0)+\gamma_{f}^{-1} \cdot \operatorname{trace}\left(\widetilde{A}_{f}(0)^{T} \cdot \widetilde{A}_{f}(0)\right) \\
& +\int_{0}^{t_{f}}\left\{e(t)^{T} \cdot\left(Q+P^{T} \cdot R^{-T} \cdot P\right) \cdot e(t)-\rho^{2}\right. \\
& \cdot e_{\mathrm{mod}}^{T} \cdot e_{\mathrm{mod}}+\left[\alpha \cdot e(t)+h_{f}(y) \cdot \widetilde{A}_{f}(t)-R^{-1}\right. \\
& \left.\cdot P \cdot e(t)+e_{\mathrm{mod}}\right]^{T} \cdot P \cdot e(t)+e(t)^{T} \cdot P \cdot[\alpha \cdot e(t) \\
& \left.+h_{f}(y) \cdot \widetilde{A}_{f}(t)-R^{-1} \cdot P \cdot e(t)+e_{\mathrm{mod}}\right]+\gamma_{f}^{-1} \\
& \cdot \operatorname{trace}\left(\dot{\widetilde{A}}_{f}(t)^{T} \cdot \widetilde{A}_{f}(t)\right)+\gamma_{f}^{-1} \cdot \operatorname{trace}\left(\widetilde{A}_{f}(t)^{T}\right. \\
& \left.\left.\cdot \dot{\vec{A}}_{f}(t)\right)\right\} d t=e(0)^{T} \cdot P \cdot e(0)+\gamma_{f}^{-1} \\
& \cdot \operatorname{trace}\left(\widetilde{A}_{f}(0)^{T} \cdot \widetilde{A}_{f}(0)\right)+\int_{0}^{t_{f}}\left\{e ( t ) ^ { T } \cdot \left[\alpha^{T} \cdot P\right.\right. \\
& \left.+P \cdot \alpha+Q+P^{T} \cdot R^{-T} \cdot P\right] \cdot e(t)-\rho^{2} \cdot e_{\bmod }^{T} \\
& \cdot e_{\text {mod }}+\left[h_{f}(y) \cdot \widetilde{A}_{f}(t)-R^{-1} \cdot P \cdot e(t)+e_{\bmod }\right]^{T} \\
& \cdot P \cdot e(t)+e(t)^{T} \cdot P \cdot\left[h_{f}(y) \cdot \widetilde{A}_{f}(t)-R^{-1} \cdot P\right. \\
& \left.\cdot e(t)+e_{\text {mod }}\right]+\gamma_{f}^{-1} \cdot \operatorname{trace}\left(\dot{\widetilde{A}}_{f}(t)^{T} \cdot \widetilde{A}_{f}(t)\right) \\
& \left.+\gamma_{f}^{-1} \cdot \operatorname{trace}\left(\widetilde{A}_{f}(t)^{T} \cdot \dot{\widetilde{A}}_{f}(t)\right)\right\} d t<e(0)^{T} \\
& \cdot P \cdot e(0)+\gamma_{f}^{-1} \cdot \operatorname{trace}\left(\widetilde{A}_{f}(0)^{T} \cdot \widetilde{A}_{f}(0)\right) \\
& +\int_{0}^{t_{f}}\left\{-\rho^{-2} e(t)^{T} \cdot P^{T} \cdot P \cdot e(t)-\rho^{2} \cdot e_{\bmod }^{T} \cdot e_{\bmod }\right. \\
& +e_{\bmod }^{T} \cdot P \cdot e(t)+e(t)^{T} \cdot P \cdot e_{\bmod }+\left[h_{f}(y)\right. \\
& \left.\cdot \widetilde{A}_{f}(t)\right]^{T} \cdot P \cdot e(t)+e(t)^{T} \cdot P \cdot h_{f}(y) \cdot \widetilde{A}_{f}(t) \\
& +\gamma_{f}^{-1} \cdot \operatorname{trace}\left(\dot{\vec{A}}_{f}(t)^{T} \cdot \widetilde{A}_{f}(t)\right)+\gamma_{f}^{-1} \\
& \left.\cdot \operatorname{trace}\left(\widetilde{A}_{f}(t)^{T} \cdot \dot{\bar{A}}_{f}(t)\right)\right\} d t=e(0)^{T} \cdot P \\
& \cdot e(0)+\gamma_{f}^{-1} \cdot \operatorname{trace}\left(\widetilde{A}_{f}(0)^{T} \cdot \widetilde{A}_{f}(0)\right)-\int_{0}^{t_{f}}[\rho \\
& \left.\cdot e_{\mathrm{mod}}-\rho^{-1} \cdot P \cdot e(t)\right]^{T} \cdot\left[\rho \cdot e_{\mathrm{mod}}-\rho^{-1} \cdot P\right. \\
& \cdot e(t)] d t \leq e(0)^{T} \cdot P \cdot e(0)+\gamma_{f}^{-1} \\
& \cdot \operatorname{trace}\left(\widetilde{A}_{f}(0)^{T} \cdot \widetilde{A}_{f}(0)\right) \text {. }
\end{aligned}
$$

This completes the proof. 


\section{Conflict of Interests}

The authors declare that there is no conflict of interests regarding the publication of this paper.

\section{Acknowledgments}

The authors would like to acknowledge the support of the Ministry of Science and Technology of the Republic of China and the former National Science Council, under Contracts NSC 100-2628-E-468-001, 101-2221-E-468-024, 102-2221-E-468-016, 102-2221-E-182-073, 103-2221-E-468009-MY2, MOST 103-2221-E-182-045, and 104-2221-E-182008-MY2; Asia University under Contracts 98-ASIA-02, 100-asia-35, and 101-asia-29; Chang Gung University; and Chang Gung Memorial Hospital, Taiwan, under Contracts CMRPD2C0052 and CMRPD2C0053.

\section{References}

[1] J. Liu and Q. Huang, "Robust $H_{\infty}$ control for switched nonlinear system with multiple delays," Mathematical Problems in Engineering, vol. 2015, Article ID 852303, 10 pages, 2015.

[2] R.-E. Precup, R.-C. David, E. M. Petriu, S. Preitl, and M.-B. Radac, "Novel adaptive charged system search algorithm for optimal tuning of fuzzy controllers," Expert Systems with Applications, vol. 41, no. 4, pp. 1168-1175, 2014.

[3] S. S. Ge and C. Wang, "Adaptive neural control of uncertain MIMO nonlinear systems," IEEE Transactions on Neural Networks, vol. 15, no. 3, pp. 674-692, 2004.

[4] T.-H. S. Li and Y.-C. Huang, "MIMO adaptive fuzzy terminal sliding-mode controller for robotic manipulators," Information Sciences, vol. 180, no. 23, pp. 4641-4660, 2010.

[5] V. Nekoukar and A. Erfanian, "Adaptive fuzzy terminal sliding mode control for a class of MIMO uncertain nonlinear systems," Fuzzy Sets and Systems, vol. 179, pp. 34-49, 2011.

[6] T. Qian and S. Miao, "Discrete-time nonlinear control of VSCHVDC system," Mathematical Problems in Engineering, vol. 2015, Article ID 929467, 11 pages, 2015.

[7] M. Erhard and H. Strauch, "Sensors and navigation algorithms for flight control of tethered kites," in Proceedings of the European Control Conference (ECC '13), pp. 1-6, Zürich, Switzerland, July 2013.

[8] V. Dobrokhodov, E. Xargay, N. Hovakimyan, I. Kaminer, C. Cao, and I. M. Gregory, "Multicriteria analysis of an $L 1$ adaptive flight control system," Proceedings of the Institution of Mechanical Engineers, Part I: Journal of Systems and Control Engineering, vol. 227, no. 4, pp. 413-427, 2013.

[9] L. Benvenuti, M. D. Di Benedetto, and J. W. Grizzle, "Approximate output tracking for nonlinear non-minimum phase systems with an application to flight control," International Journal of Robust and Nonlinear Control, vol. 4, no. 3, pp. 397-414, 1994.

[10] A. Isidori and C. I. Byrnes, "Output regulation of nonlinear systems," IEEE Transactions on Automatic Control, vol. 35, no. 2, pp. 131-140, 1990.

[11] S. A. Al-Hiddabi, "Design of a flight control system for a nonminimum phase 5 DOF aircraft model," in Proceedings of the 11th Mediterranean Conference on Control and Automation (MED '03), pp. 18-20, Rhodes Island, Greece, June 2003.

[12] Y.-Z. Chang and Z.-R. Tsai, "Supervised adaptive control of unknown nonlinear systems using fuzzily blended time-varying canonical model," in New Trends in Applied Artificial Intelligence, vol. 4570 of Lecture Notes in Computer Science, pp. 464472, Springer, Berlin, Germany, 2007.

[13] Q. Gao, G. Feng, Y. Wang, and J. Qiu, "Universal fuzzy controllers based on generalized T-S fuzzy models," Fuzzy Sets and Systems, vol. 201, pp. 55-70, 2012.

[14] E. P. Klement and R. Mesiar, "A concept of universal fuzzy integrals," in Proceedings of the Annual Meeting of the North American Fuzzy Information Processing Society (NAFIPS '12), pp. 1-4, Berkeley, Calif, USA, August 2012.

[15] Q. Gao, X.-J. Zeng, G. Feng, and Y. Wang, "Universal fuzzy models and universal fuzzy controllers based on generalized T-S fuzzy models," in Proceedings of the IEEE International Conference on Fuzzy Systems (FUZZ '12), pp. 1-6, Brisbane, Australia, June 2012.

[16] S. S. Sastry and A. Isidori, "Adaptive control of linearizable systems," IEEE Transactions on Automatic Control, vol. 34, no. 11, pp. 1123-1131, 1989.

[17] H.-J. Uang and B.-S. Chen, "Robust adaptive optimal tracking design for uncertain missile systems: a fuzzy approach," Fuzzy Sets and Systems, vol. 126, no. 1, pp. 63-87, 2002.

[18] A. Stoorvogel, The $H_{\infty}$ Control Problem: A State Approach, Prentice-Hall, Englewood Cliffs, NJ, USA, 1992.

[19] T. Basar and G. J. Olsder, Dynamic Noncooperative Game Theory, Academic Press, London, UK, 1982.

[20] I. Rhee and J. L. Speyer, "A game theoretic approach to a finitetime disturbance attenuation problem," IEEE Transactions on Automatic Control, vol. 36, no. 9, pp. 1021-1032, 1991.

[21] J. Lévine, "Automatic flight control systems," in Analysis and Control of Nonlinear Systems, Mathematical Engineering, pp. 295-315, Springer, Berlin, Germany, 2009. 


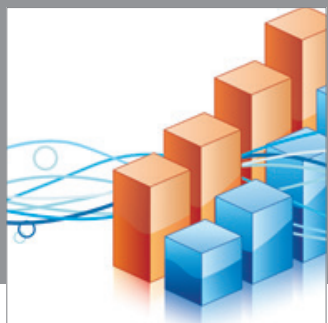

Advances in

Operations Research

mansans

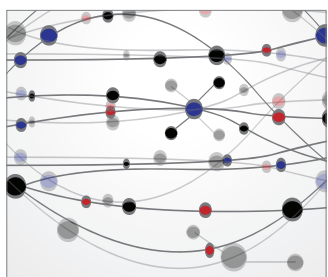

The Scientific World Journal
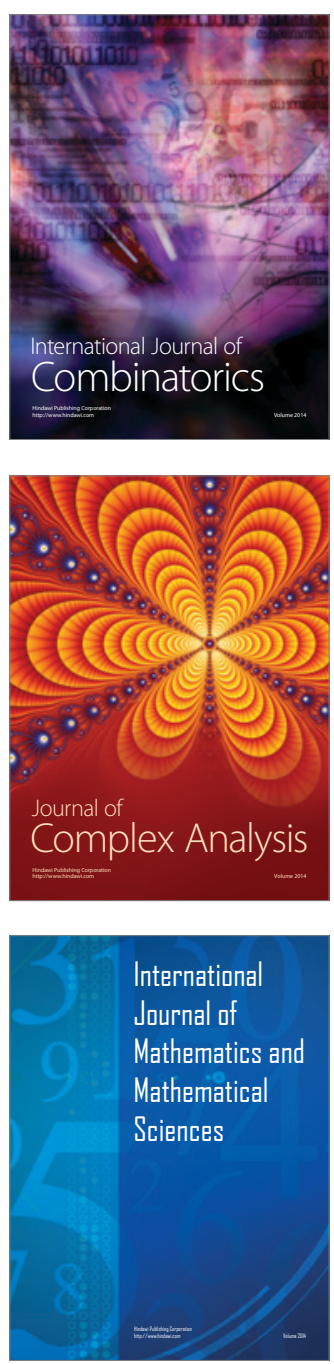
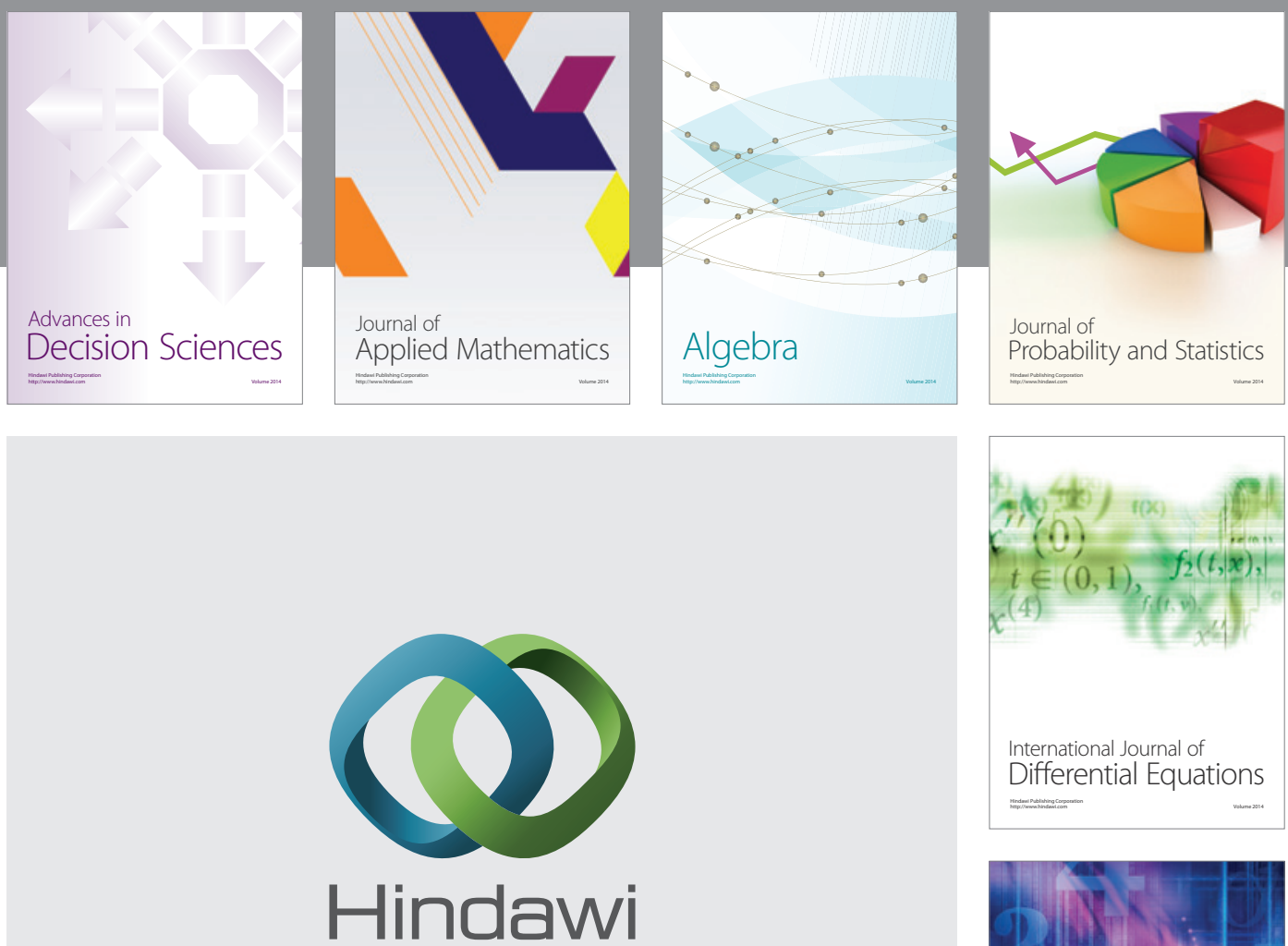

Submit your manuscripts at http://www.hindawi.com
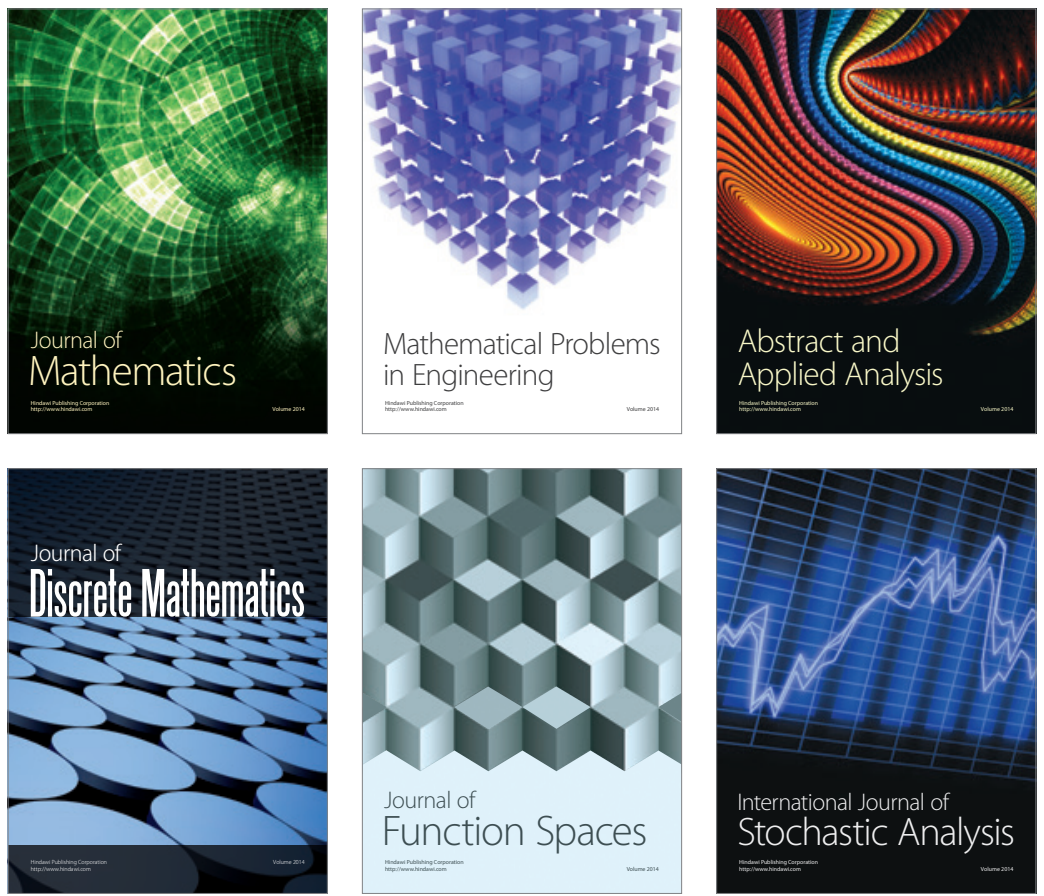

Journal of

Function Spaces

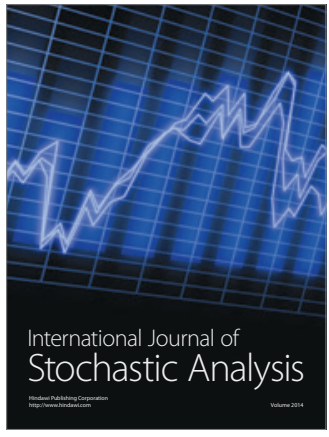

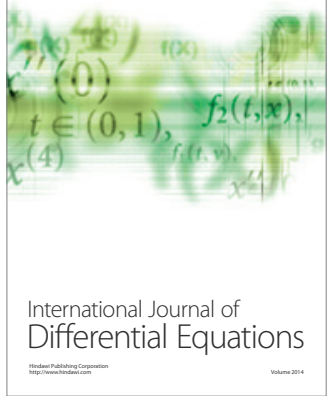
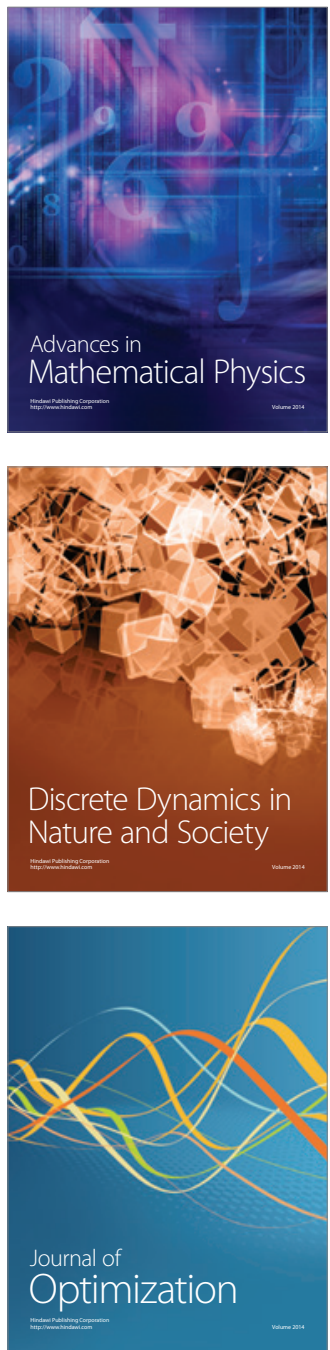